\title{
Electromyography of the Mandibular Postural Position in the Rhesus Monkey (Macaca mulatta)
}

\author{
JAMES A. MCNAMARA, JR. \\ Department of Anatomy and Center for Human Growth and Development, \\ University of Michigan, Ann Arbor, Michigan 48104, USA
}

The mandibular postural position is attained when all of the muscles involved in mandibular movement show no other activity than that which is necessary to hold the mandible in a balanced position against gravity. Mandibular position is influenced by the fusimotor system through psychic input and through such stimuli from peripheral receptors as those of the tem. poramandibular joint, periodontal ligaments, gingiva, tongue, and palate. Mandibular position also is related to the maintenance of the pharyngeal airway. The postural position of the mandible in man is maintained by tonic activity in the elevator musculature opposing gravitational forces. The purpose of this study was to determine the relative participation of the orofacial muscles of the rhesus monkey in the maintenance of mandibular posture.

Thirty-three Macaca mulatta, aged six months to eight years, were monitored in 113 electro. myographic recording sessions. Bipolar needle electrodes were placed in the anterior and posterior portions of the temporal muscle, the an terior portion of the superior head of the mass eter muscle, the orbicularis oris muscle, the two heads of the lateral pterygoid muscle, and in the suparhyoid muscle group. Posture, oral reflexes, and random jaw movement were studied while each animal was isolated in a sound.

This study was supported in part by USPHS Grants HD-02272 and DE-03610.

Received for publication September 17, 1973.
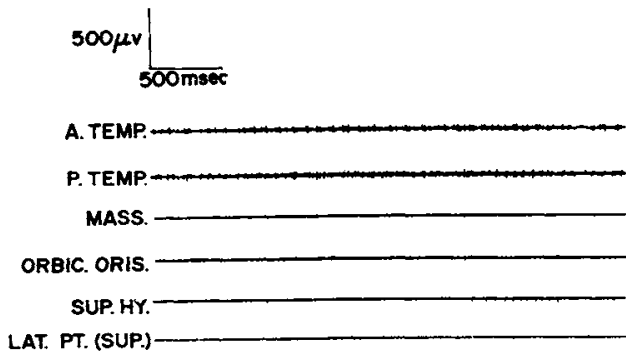

FIG 1.-Mandibular postural activity. Tonic discharges observed in anterior and posterior portions of temporal muscle (recording speed $100 \mathrm{~mm} /$ second) .

J Dent Res July-August 1974, Vol 53 No. 4

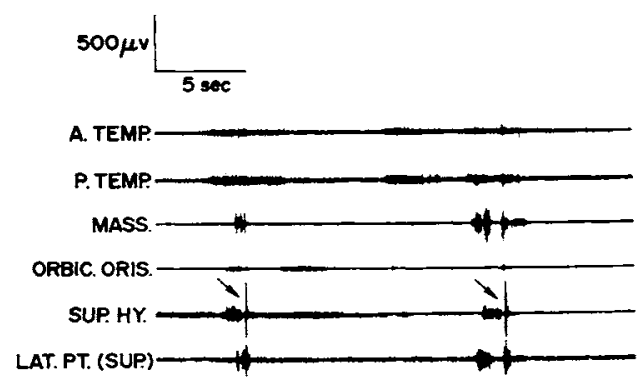

FIG 2.-Overview of postural activity. Two salivary swallows are indicated by arrows (re. cording speed $5 \mathrm{~mm} /$ second).

proof, electrically shielded chamber. Further technical details are reported elsewhere (McNAMARA, Am J Anat, 138: 197-206, 1973) .

The temporal muscle (particularly the anterior portion) was the most active muscle during postural recordings (Fig 1 and 2). Continuous tonic discharges of this muscle were observed nearly always. The masseter muscle usually showed intermittent activity rather than continuous tonic firings (Fig 2). The inferior head of the lateral pterygoid muscle usually was inactive during postural recordings; whereas, occasional discharges were observed in the superior head, which were repeated at a rate that varied considerably in the different animals. Discharges from the suprahyoid muscle group (anterior digastric, mylohyoid, and geniohyoid), which is an activity presumably associated in part with the support of the hyoid bone, were observed frequently.

The results of this study suggest that the temporal muscle in the rhesus monkey has a primary role in the maintenance of the mandibular postural position. The masseter muscle, the superior head of the lateral pterygoid muscle, and the suprahyoid muscle group also show spontaneous electromyographic activity when the mandible is at rest. The amount of this activity varies among muscles and also within individual muscles.

The author thanks M. C. McBride for her technical assistance. 